\title{
Acknowledgment of Principal and Ad Hoc Reviewers (2021)
}

\section{Principal Reviewers}

The Editor gratefully acknowledges the following persons who served as Principal reviewers for Personality Disorders: Theory, Research, and Treatment, reviewing a minimum of 3 manuscripts in the past year.

\begin{tabular}{|c|c|c|c|}
\hline Skye Fitzpatrick & Thomas R. Kwapil & Joshua Oltmanns & $\begin{array}{l}\text { Lori N. Scott } \\
\text { Chelsea Sleep }\end{array}$ \\
\hline Luke Hyde & Jessica L. Maples-Keller & Jessica R. Peters & Colin E. Vize \\
\hline
\end{tabular}

\section{Ad Hoc Reviewers}

The Editor gratefully acknowledges the following persons who reviewed manuscripts submitted to Personality Disorders: Theory, Research, and Treatment in the past year. Reviewers who reviewed manuscripts under the supervision of a primary reviewer are denoted with an asterisk.

$\begin{array}{ll}\begin{array}{l}\text { Jaime Anderson } \\ \text { Arnoud Arntz }\end{array} & \begin{array}{l}\text { Nicholas Eaton } \\ \text { Emily Edwards* }\end{array} \\ \begin{array}{l}\text { Julia C. Babcock } \\ \text { Bo Bach }\end{array} & \begin{array}{l}\text { Sarah Fischer } \\ \text { Mitja Back }\end{array} \\ \text { Jichael Bagby Foster } \\ \text { Arielle Baskin-Sommers } & \text { Lindsay Fullham* } \\ \text { Kendra Becker } & \text { Kristin Gainey } \\ \text { Joseph Beeney } & \text { Carlo Garofalo } \\ \text { Sune Bo } & \text { Marianne Goodman } \\ \text { Robert Bornstein } & \text { Brin F.S. Grenyer } \\ \text { Michael Boudreaux } & \text { Sarah A. Griffin } \\ \text { Cassie Brandes } & \\ \text { Ryan W. Carpenter } & \text { Lauren Haliczer* } \\ \text { Lois Choi-Kain } & \text { Stephen Hart } \\ \text { Lee Anna Clark } & \text { Stephanie Hruza* } \\ \text { Matthew Constantinou } & \text { Nathan Hudson } \\ \text { Christopher C. Conway } & \text { Steven Huprich } \\ \text { Cristina Crego } & \text { Joost Hutsebaut } \\ \text { Michael Crowe } & \text { Benjamin N. Johnson } \\ \text { Matt DeLisi } & \text { Keanan Joyner } \\ \text { Hilary L. DeShong } & \\ \text { Linda Dimeff } & \text { David Kealy } \\ \text { Eva R. Kimonis }\end{array}$

*Denotes a co-reviewer.

Justin A. Lavner
Majse Lind
Adam Mann*
Jessica L. Maples-Keller
David Marcus
Courtney Mason*
Shelley McMain
Conal Monaghan
Bryan Neo*
Craig Neumann
Max Owens
Anthony Pinto

Whitney R. Ringwald Michael Roche Craig Rodriguez-Seijas Anthony C. Ruocco

\section{Randall T. Salekin}

Shannon Sauer-Zavala Edward Selby

Matthew Shane

Carla Sharp
Rebecca Shiner

Sebastian Simonsen

Robert J. Snowden

Shauna Solomon-Krakus

Kasey Stanton

Amanda Uliaszek

Salome Vanwoerden

Sonya Varma*

Lize Verbeke

Edelyn Verona

Glenn D. Walters

Bruce Wampold

Ashley Watts

Mark Waugh

Michael Wheaton

Andrew White*

Catherine Winspur

Maureen Zalewski

Mark Zimmerman

Johannes Zimmermann 